\title{
Fecal transplant clinical trials for Clostridioides difficile: an interview with Sahil Khanna
}

\author{
Sahil Khanna*,1 \\ ${ }^{1}$ Associate Professor of Medicine, Division of Gastroenterology \& Hepatology, Mayo Clinic, 200 First St SW, Rochester, MN \\ 55905, USA \\ *Author for correspondence: Khanna.Sahil@mayo.edu
}

${ }^{\prime}{ }^{C}$. difficile is now the most common infection in hospitals in the US, having surpassed methicillin-resistant Staphylococcus aureus and vancomycin-resistant Enterococcus. In the last 2 decades, the proportion of $\mathrm{CDI}$ in the community has been increasing. It is now estimated that $40 \%$ of all CDI cases are community acquired."

This interview was conducted by Atiya Henry, Commissioning Editor of Future Microbiology.

Sahil Khanna is an Associate Professor of Medicine in the Division of Gastroenterology and Hepatology at Mayo Clinic, Rochester (MN, USA). He currently directs the Comprehensive Gastroenterology Interest group, Clostridioides difficile clinic, Fecal Microbiota Transplantation program and C. difficile related clinical trials at Mayo Clinic. He completed Medical School at the All India Institute of Medical Sciences, New Delhi; followed by Post Doctoral Research at University of California, San Diego (CA, USA); residency in Internal Medicine and Fellowship in Gastroenterology and Hepatology at Mayo Clinic, before joining the Faculty. He also completed Masters in Clinical and Translational Sciences during his fellowship. His research and clinical interests include epidemiology, outcomes and emerging therapeutics for $C$. difficile infection, an arena in which he has had numerous publications and presentations. He has over 100 peer-reviewed publications, serves as reviewer, is on the editorial board of several journals and has won numerous awards.

Keywords: antibiotics • Clostridioides difficile • epidemiology • fecal microbiota transplantation • microbiota restoration therapies

\section{Your work is focused on therapy for CDI. Can you tell me about your research \& what first drew you to this area of work?}

I was first involved in research on Clostridioides diffcile infections (CDI) in 2008 with initial studies on the epidemiology and outcomes of $C$. difficile infection under the guidance of my mentor, Darrell Pardi. My research in $C$. difficile ranges from epidemiology of community and hospital-acquired infection, outcomes of CDI, novel risk factors for adverse outcomes, management of CDI in inflammatory bowel disease (IBD), testing strategies for $\mathrm{CDI}$, microbiome predictors of CDI outcomes, outcomes of microbial replacement therapies for CDI and clinical trials for CDI therapies. Excellent mentorship and the available resources for research at Mayo Clinic drew me toward this research.

\section{Could you tell me a bit more about the epidemiology of CDI \& the current treatment guidelines?}

We used to consider CDI as a hospital-acquired illness with primary risk factors being age of over 65 years, antibiotic exposure and comorbid conditions. With the advent of better testing strategies for CDI (namely PCR-based testing), the incidence of CDI has increased due to more sensitive testing and increased awareness of the infection. C. difficile is now the most common infection in hospitals in the US, having surpassed methicillin-resistant Staphylococcus aureus and vancomycin-resistant Enterococcus. In the last 2 decades, the proportion of CDI in the community has been increasing. It is now estimated that $40 \%$ of all CDI cases are community acquired. These patients are

Future $\because$ Medicine 
often younger, have fewer comorbidities, and one in five patients with community-acquired CDI do not have prior antibiotic exposure [1]. Recent data have shown that in addition to the increase in primary CDI, the risk of recurrent CDI has been increasing exponentially, with one study estimating a $189 \%$ increase in incidence of recurrent CDI in a little over a decade, compared with $43 \%$ increase in the incidence of primary CDI [2].

Treatment guidelines for CDI have changed recently with the evolution of data regarding treatment success and failure, availability of vancomycin in a liquid form (decreased cost), the approval of fidaxomicin and the availability of microbiome-based therapies for management of recurrent CDI. According to recent guidelines, the recommended treatment for first infection (mild-moderate or severe) is a 10-day course of vancomycin or fidaxomicin. Metronidazole is no longer recommended as a first-line treatment except in situations of mildmoderate CDI when vancomycin or fidaxomicin are not available. Treatment of first recurrence is based on the treatment used for the first infection. In general, the treatment used for the first infection should not be used for the first recurrence. Options for first recurrence generally include a vancomycin taper or fidaxomicin standard 10-day course [3]. Although not a part of current treatment guidelines, one-time intravenous bezlotoxumab (monoclonal anti-toxin B IgG) in addition to antibiotic therapy has been shown to reduce recurrent CDI in a subset of patients (those with high risk of recurrence) [4]. Treatment options for second or subsequent recurrence include vancomycin taper, fidaxomicin, vancomycin followed by rifaximin or fidaxomicin and the most effective therapy being vancomycin or fidaxomicin followed by fecal microbiota transplantation [3].

\section{The field is now looking toward MRTs \& FMT for treatment of CDI. Could you tell us a bit about the theory behind FMT \& how it works?}

Among microbiota restoration therapies (MRTs), fecal microbiota transplantation (FMT) is the most common form of MRT used to treat multiple recurrent CDI. The human gut microbiome is suspected to harbor over 1000 different species and 100 trillion bacteria in the colon. The healthy flora is disrupted by antibiotics or other risk factors for $\mathrm{CDI}$ and a niche for the $C$. difficile spore is created. $C$. difficile spores generate vegetative forms and produce toxins that lead to diarrhea. Vancomycin and fidaxomicin have activity against the vegetative forms but not the spore forms of $C$. difficile. Stopping antibiotics that treat CDI leads to a chance of recurrence, which increases with every episode of CDI. The risk of recurrent CDI after a third episode is $60 \%$ or higher. A microbiota sample obtained from a healthy donor is likely to prevent future CDI through a number of mechanisms such as competitive exclusion of the $C$. difficile spores and restoration of the bile acid profiles, among others. The reported success rates (prevention of future recurrences) from FMT are over $80 \%$, significantly higher than success rates for antibiotics [5].

\section{Could you tell me about your most recent clinical trial investigating FMT as a therapy for CDI?}

Our group has been a part of several clinical trials for microbiome replacement therapies. We have been involved in running Phase I through to Phase III clinical trials. Two of the products that have completed Phase III trials are RBX2660 and SER-109.

RBX2660 is a microbiota suspension of intestinal microbes derived from human stool. Results from a Phase II blinded trial of RBX2660 for recurrent CDI were positive. This was a randomized, double-blind, placebo-controlled Phase II trial, enrolling adults with two or more CDI recurrences to receive: two doses of RBX2660; two doses of placebo or one dose of RBX2660 followed by one dose of placebo. The primary end point was not met but one RBX2660 dose was superior to placebo and the overall efficacy (including open-label response) for patients that received at least one dose of RBX2660 was $88.8 \%$ [6].

We also recently completed a prospective, multi-center, randomized, double-blinded, placebo-controlled Phase III study of RBX2660. Patients who had at least one recurrence after a primary episode and had completed at least one round of oral antibiotic therapy or had at least two episodes of severe CDI resulting in hospitalization within the last year were eligible for the study. The results from this trial are eagerly awaited.

SER-109 is derived from human donor stool specimens treated with ethanol to eliminate pathogens. The resulting spores are fractionated and encapsulated for oral delivery as SER-109. A Phase I open-label study demonstrated SER-109 successfully prevented recurrent CDI and had a favorable safety profile [7]. A Phase II, multi-center, randomized, double-blind, placebo-controlled study with two treatment arms (SER-109 or placebo), failed to demonstrate a benefit of SER-109 over placebo to prevent recurrent CDI [8]. A Phase III, multicenter, randomized, double-blind, placebo-controlled, parallel-group study of the safety, tolerability and efficacy of SER-109 versus 
placebo in adult subjects with recurrent CDI was recently completed. This study was designed to demonstrate the superiority of SER-109 versus placebo to reduce recurrent CDI. Results are eagerly awaited.

\section{What are some of the biggest challenges in FMT \& in getting it US FDA approved?}

There are several challenges for FMT to get FDA approved. The first challenge includes the availability of highquality data on efficacy and safety (from placebo-controlled trials). Second, there is a pressing need to standardize donor health screening, donor blood testing and stool testing for infections to establish safety. Third, there is a need for standardization of standard operating processes for preparation, storage and embargo of donor stool for use for FMT.

\section{In late 2019, two patients developed drug-resistant infections after FMT, with one sadly passing away. What were the suspected causes behind this \& what action is being taken?}

The case reports of the two patients, who developed extended spectrum beta-lactamase (ESBL)-producing Escherichia coli infections, had the same bacterial strain as the donor. These two patients were immunocompromised and received FMT through two different research studies. The material was obtained from a donor at a time when the stool samples were not being tested for ESBL-producing E. coli. There were some patients who received donor material from the same donor for treatment of recurrent CDI, who later were colonized with ESBL-producing $E$. coli but fortunately did not get a systemic infection [9]. These data suggest that asymptomatic healthy individuals may be colonized by multidrug-resistant bacteria and the FDA recommends that testing for such bacteria should be part of screening protocols for stool donors.

\section{I understand that you also investigate antibiotics for CDI. How do they compare with FMT?}

There have been narrow spectrum antibiotics studied for the treatment of CDI. Fidaxomicin was approved many years ago for management of CDI and it has an advantage over vancomycin as it causes fewer recurrences compared with vancomycin [5]. Surotomycin demonstrated noninferiority to vancomycin for CDI clinical response but did not demonstrate superiority to vancomycin for clinical response over time or sustained clinical response rate. Cadazolid did not achieve its primary end point of noninferiority to vancomycin for clinical cure in one of the two Phase III CDI trials. Both cadazolid and surotomycin are not being further developed to treat CDI.

Ridinilazole is a targeted-spectrum antimicrobial that is currently in Phase III trials for treatment of acute CDI. In a Phase II, randomized, double-blinded, noninferiority study, ridinilazole exhibited statistical superiority to vancomycin for sustained clinical response through a reduction in CDI recurrence [10].

Narrow spectrum antibiotic therapies for CDI would reduce recurrences after an initial infection but would probably not replace FMT, which I think would remain the treatment of choice to prevent recurrent CDI after multiple CDI episodes.

\section{What is your expectation for research into therapies for CDI over the next 10 years?}

Over the next 10 years, I'd expect there to be ongoing research in order to obtain a better understanding of both microbiome-based risk factors for recurrent CDI and the epidemiology of primary and recurrent CDI. We hope to continue to run clinical trials for approval of additional narrow spectrum antibiotics for active CDI. I'd also expect there to still be ongoing and completed clinical studies, that help to have better defined evidence-based treatments for first CDI recurrence. Modeling and clinical algorithm-based studies should be done for monoclonal antibodies such as bezlotoxumab to be better placed in the treatment algorithm for CDI. We will continue to develop standardized microbiome-based therapies to prevent recurrent CDI, including those that are derived from donor stool and those that are synthetically prepared defined microbial consortia. There should also be studies completed that define a role for tailored probiotics, such as non-toxigenic C. difficile. At last, efforts are underway for development of a vaccine to prevent primary CDI.

\section{Disclaimer}

The opinions expressed in this interview are those of the interviewee and do not necessarily reflect the views of Future Medicine Ltd. 


\section{Financial \& competing interests disclosure}

S Khanna has received research support from Rebiotix, Inc. (a Ferring company) and Vedanta. The author has no other relevant affiliations or financial involvement with any organization or entity with a financial interest in or financial conflict with the subject matter or materials discussed in the manuscript apart from those disclosed.

No writing assistance was utilized in the production of this manuscript.

\section{References}

1. Khanna S, Pardi DS, Aronson SL et al. The epidemiology of community-acquired C. difficile infection: a population-based study. Am. J. Gastroenterol. 107(1), 89-95 (2012).

2. Ma GK, Brensinger CM, Wu Q, Lewis JD. Increasing incidence of multiply recurrent $C$. difficile infection in the USA: a cohort study. Ann. Intern. Med. 167(3), 152-158 (2017).

3. McDonald LC, Gerding DN, Johnson S et al. Clinical practice guidelines for C. difficile infection in adults and children: 2017 update by the Infectious Diseases Society of America (IDSA) and Society for Healthcare Epidemiology of America (SHEA). Clin. Infect. Dis. 66(7), e1-e48 (2018).

4. Gerding DN, Kelly CP, Rahav G et al. Bezlotoxumab for prevention of recurrent $C$. difficile infection in patients at increased risk for recurrence. Clin. Infect. Dis. 67(5), 649-656 (2018).

5. Cho JM, Pardi DS, Khanna S. Update on treatment of C. difficile infection. Mayo Clin. Proc. 95(4), 758-769 (2020).

6. Dubberke ER, Lee CH, Orenstein R, Khanna S, Hecht G, Gerding DN. Results from a randomized, placebo-controlled clinical trial of a RBX2660-a microbiota-based drug for the prevention of recurrent clostridium difficile infection. Clin. Infect. Dis. 67(8), 1198-1204 (2018).

7. Khanna S, Pardi DS, Kelly CR et al. A novel microbiome therapeutic increases gut microbial diversity and prevents recurrent $C$. difficile infection. J. Infect. Dis. 214(2), 173-181 (2016).

8. McGovern BH, Ford CB, Henn MR et al. SER-109, an investigational microbiome drug to reduce recurrence after $C$. difficile infection: lessons learned from a Phase II trial. Clin. Infect. Dis. doi:10.1093/cid/ciaa387 (2020) (Epub ahead of print).

9. DeFilipp Z, Bloom PP, Torres Soto M et al. Drug-resistant E. coli bacteremia transmitted by fecal microbiota transplant. N. Engl. J. Med. 381(21), 2043-2050 (2019).

10. Vickers RJ, Tillotson GS, Nathan R et al. Efficacy and safety of ridinilazole compared with vancomycin for the treatment of $C$. difficile infection: a Phase II, randomised, double-blind, active-controlled, noninferiority study. Lancet Infect. Dis. 17(7), 735-744 (2017). 